\title{
Vida a fatiga de uniones soldadas mediante PAW de aceros microaleados al boro
}

\author{
Fatigue life of PAW welded joints of high \\ strength microalloyed boron steels
}

Horacio Carlos Nadale ${ }^{1}$, Hernán Gabriel Svoboda ${ }^{1,2}$,

\author{
${ }^{1}$ Grupo de Tecnología de la Soldadura y Comportamiento Mecánico de Materiales Metálicos - GTSyCM3 - CP: 1127, \\ Av. Las Heras 2214, CABA, Buenos Aires, Argentina. \\ e-mail: horaciocnadale@hotmail.com \\ ${ }^{2}$ CONICET, CP: C1425FQB, Godoy Cruz 2290 - CABA, Buenos Aires, Argentina. \\ e-mail: hsvobod@fi.uba.ar
}

\section{RESUMEN}

En el último tiempo los aceros microaleados al boro se han presentado en la industria automotriz como una alternativa para la disminución de peso de estas estructuras. Típicamente, se emplean en condición de temple y revenido (TR) con resistencias a la tracción entre 800 y $1300 \mathrm{MPa}$ en elementos estructurales como parantes, columnas o refuerzos. Asimismo, los aceros Dual-Phase (DP) también ocupan un lugar de relevancia en esta industria, dada la particular combinación de elevada resistencia y aceptable ductilidad, junto con una gran capacidad de absorción de energía. La estructura del automóvil es primordialmente de construcción soldada, por tanto, la soldadura de estos materiales es un aspecto trascendental. El proceso PAW presenta aspectos que lo vuelven una opción interesante para estas aplicaciones. Asimismo, la vida a la fatiga de estas uniones soldadas es un aspecto de fundamental importancia en este tipo de elementos. El objetivo de este trabajo fue evaluar la vida a la fatiga de uniones soldadas ejecutadas mediante el proceso PAW de aceros microaleados al boro en chapa fina $(1 \mathrm{~mm})$ con $900 \mathrm{MPa}$ de resistencia a la tracción, en dos condiciones microestructurales: temple y revenido (TR) y dual-phase (DP). A tal fin, se obtuvieron ambas condiciones microestrucutrales mediante tratamientos térmicos intercríticos y de temple y revenido. Posteriormente, mediante el proceso mencionado, se soldaron cupones de 100x100mm, caracterizándose microestructural y mecánicamente (perfiles de dureza y ensayos de tracción) las uniones soldadas. Finalmente, para ambos casos (PAW+TR y $\mathrm{PAW}+\mathrm{DP}$ ), se ensayaron a fatiga probetas en flexión en cuatro puntos pulsante, obteniéndose las curvas S-N. Se observó que las probetas PAW+DP presentaron superior vida a fatiga que las PAW+TR. Este resultado estaría asociado a la evolución microestructural que experimentan ambos materiales.

Palabras clave: Soldadura PAW, aceros microaleados al Boro, DP, fatiga.

\section{ABSTRACT}

In the last years microalloyed boron steels have been adopted in the automotive industry to achieve weight reductions. Namely, they are used as load-bearing elements such as pillars and reinforcements, in a quenched and tempered (QT) condition with tensile strength ranging from 800 to $1300 \mathrm{MPa}$. Dual-Phase (DP) steels have also been introduced in this industry given their good combination of high strength and ductility, coupled with good energy absorption characteristics. The automobile is largely a welded steel frame construction. Thus, the weldability of these advanced high strength steels is a key aspect. The Plasma Arc Welding process (PAW) presents features that make it suitable for these applications. Furthermore, the mechanical response, specifically the fatigue behavior of weldments is crucial for this kind of applications. In this manner, the purpose of this work was to study the fatigue response of PAW welded joints of $1 \mathrm{~mm}$ high strength (900 MPa) microalloyed boron steels sheets in two microstructural conditions: quenched and tempered (QT) and dualphase (DP). Intercritical and quenching and tempering heat treatments were performed. Coupons of 100x100mm were welded by PAW and characterized. Finally, by means of pulsating 4-point bending fatigue tests, fatigue curves were obtained for both types of welded joints (PAW+TR and PAW+DP). A superior fatigue life was measured for de PAW+DP samples. This observation could be related to the different micro- 
structural evolution experienced in the heat affected zone of this two materials.

Keywords: Weld, fatigue, microalloyed boron steels, DP.

\section{INTRODUCCIÓN}

La preocupación medioambiental ha promovido la sanción de legislaciones en diversos países del mundo en el marco de las cuales las industrias automotrices se han inclinado por el diseño y fabricación de estructuras más livianas y seguras [1]. Como consecuencia de esto han surgido los Aceros Avanzados de Alta Resistencia (AHSS), destacándose los aceros de fase dual (Dual Phase, DP) y los aceros microaleados al boro (BMS).

Los aceros DP se componen principalmente de islas de martensita $\left(\alpha^{\prime}\right)$ embebidas en una matriz ferrítica $(\alpha)$. Resultado de la compleja interacción de sus fases, estos aceros presentan elevada resistencia, apreciable ductilidad, buena conformabilidad soldabilidad y absorción de energía [2]. Se logran por tratamientos térmicos intercrítico en gran variedad de materiales, entre ellos, aceros microaleados al boro [3]. Estos últimos se utilizan tradicionalmente en condición de temple y revendido (TR), aplicándose la tecnología de Hot Forming, logrando reducir la recuperación elástica durante el proceso de conformado y alcanzar resistencias entre los 800 y $1300 \mathrm{MPa}[4,5]$.

Las uniones mediante procesos de soldadura por fusión constituyen en esta industria una temática primordial dado que la estructura del automóvil es principalmente soldada. Por tanto, la soldadura de aceros AHSS es un aspecto relevante, siendo de particular interés procesos como el GMAW, el de Punto por Resistencia (RSW), Láser (LW) y el PAW (Plasma Arc Welding). La elevada densidad de energía del arco y la no utilización de material de aporte hacen del proceso PAW una opción atractiva para la fabricación de componentes estructurales de vehículos automotores [5].

La alteración microestructural que sucede en la Zona Afectada por el Calor (ZAC) de uniones soldadas de aceros AHSS, particularmente la formación de zonas blandas, tiende a disminuir la eficiencia de la junta conforme aumenta la resistencia de los materiales base a unir $[4,6]$.

Los componentes estructurales vehiculares están sujetos a cargas que fluctúan en el tiempo, consecuentemente son susceptibles a fallas por fatiga [4]. Así, es importante considerar no solo la respuesta estática, sino también el comportamiento a fatiga de las uniones soldadas. Concentradores geométricos de tensión, defectos de soldadura, tensiones residuales de soldadura y aspectos de índole metalúrgico, definirán la vida a fatiga de las juntas soldadas [7]. En este marco, la información disponible en relación a la vida a fatiga de uniones soldadas mediante diversos procesos en AHSS es limitada, más aún, en aceros microaleados al boro. Por tanto, el propósito del presente trabajo es evaluar comparativamente el comportamiento a la fatiga de uniones soldadas mediante el proceso PAW en un acero microaleado al boro de alta resistencia, en dos condiciones microestructurales: TR y DP.

\section{MATERIALES Y MÉTODOS}

Como material de partida se utilizó una chapa comercial de $1 \mathrm{~mm}$ de espesor de acero microaleado al boro (MAB). Mediante Espectrometría de Emisión Óptica (OES) se determinó la composición química del material de partida.

A fin de lograr las dos condiciones microestructurales de interés, martensítica revenida (TR) y dualphase (DP), ambas con la misma dureza $(300 \mathrm{HV})$, sobre muestras de $50 \times 100 \mathrm{~mm}^{2}$ se practicaron dos tipos de tratamientos térmicos: temple y revenido y temple intercrítico, respectivamente. El temple se logró austenizando a una temperatura de $875^{\circ} \mathrm{C}$ durante 10 minutos y enfriando en agua corriente a temperatura ambiente y posteriormente se realizó el revenido durante 30 minutos a $475^{\circ} \mathrm{C}$. El tratamiento térmico de temple intercrítico se llevó a cabo a $750^{\circ} \mathrm{C}$ por un período de 10 minutos, seguido de enfriamiento en agua. Los materiales base así logrados se caracterizaron microestructural y mecánicamente mediante microscopía óptica (LM) y electrónica de barrido (SEM), microdureza Vickers (HV1) y ensayo de tracción.

Las muestras tratadas térmicamente de ambas condiciones se soldaron a tope en forma mecanizada mediante el proceso de soldadura PAW, formando cupones de $100 \times 100 \mathrm{~mm}^{2}$, obteniéndose dos tipos de probetas, identificadas como: PAW+TR y PAW+DP. En la Tabla 1 se indican los parámetros de soldadura empleados y en la Figura 1 el montaje experimental utilizado. Las probetas así soldadas se caracterizaron microestructural y mecánicamente mediante microscopía óptica, SEM, perfiles de microdureza y ensayos de tracción transversales.

Finalmente, se realizaron ensayos de fatiga mediante flexión pulsante en cuatro puntos $(\mathrm{R}=0,1$ $20 \mathrm{~Hz}$ ) a fin de construir curvas de fatiga de los dos tipos de juntas PAW+TR y PAW+DP. Las curvas de fatiga de los materiales base no fueron evaluadas porque el interés fue evaluar la respuesta de diferentes condi- 
ciones microestructurales ante el un mismo proceso de soldadura.

Tabla 1: Parámetros de soldadura HI (Heat Input $)=\eta_{\mathrm{a}}$ V I / S.

\begin{tabular}{l|l|l|l|l|l|l}
\hline $\mathbf{I}[\mathbf{A}]$ & $\mathbf{V}[\mathbf{V}]$ & $\mathbf{S}[\mathbf{M M} / \mathbf{S}]$ & GAS (PR/PL) & $\mathbf{Q}[\mathbf{L} / \mathbf{M I N}]$ & $\boldsymbol{\eta}_{\mathbf{a}}$ & HI[J/MM] \\
\hline 50 & 13,5 & 7,7 & Ar/Ar-5H2 & $0,8 / 20$ & 0,60 & 53 \\
\hline \multicolumn{6}{c}{ Ángulo de inclinación de la torcha: $10^{\circ}$ - empujando } \\
\hline \multicolumn{6}{l}{ - Retroceso del electrodo: $1 \mathrm{~mm}$} \\
\hline \multicolumn{6}{l}{ Distancia tobera-pieza: $2 \mathrm{~mm}$} \\
\hline
\end{tabular}

I: corriente de soldadura, V: tensión de arco, S: velocidad de soldadura, PR: gas de protección, PL: gas de plasma, Q: caudal, na: rendimiento térmico.

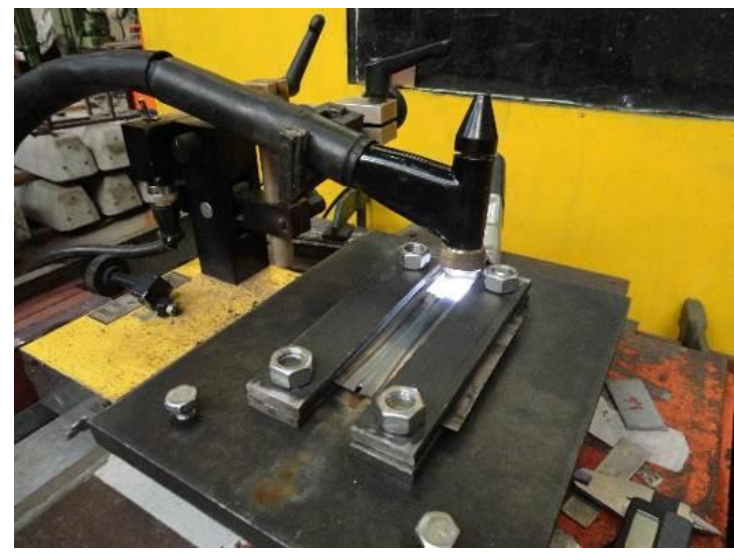

Figura 1: Montaje experimental para soldadura PAW.

La Figura 2 muestra un esquema de la forma en que se obtuvieron las probetas para los ensayos mecánicos.
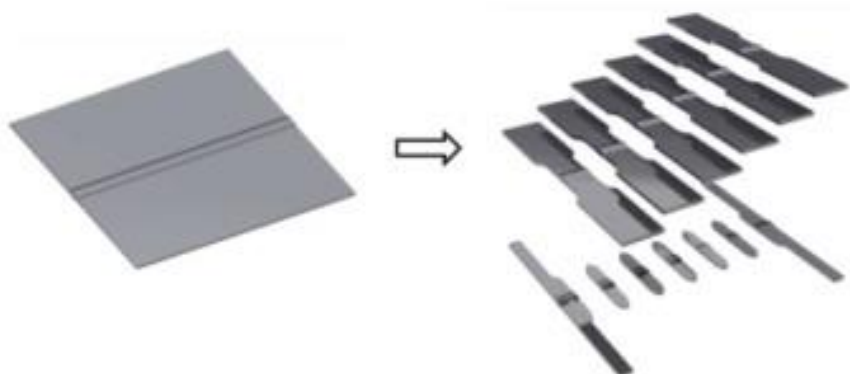

Figura 2: Esquema de extracción de probetas para ensayos mecánicos.

La longitud de los apoyos superiores e inferiores fue de 80 y $40 \mathrm{~mm}$, respectivamente. Como criterio de vida infinita se tomó $10^{7}$ ciclos. Las superficies de fractura de las probetas ensayadas fueron caracterizadas mediante microscopía óptica y electrónica de barrido (SEM), analizando la ubicación de la fractura y las características topográficas.

\section{RESULTADOS}

La Tabla 1 presenta el análisis químico de la aleación estudiada. Se trata de un acero con un contenido medio de carbono, aleado al Mn con 30ppm de Boro. 
Tabla 2: Composición química (wt\%).

\begin{tabular}{l|l|l|l|l|l|l}
\hline $\mathbf{C}$ & Mn & SI & P & S & B & Fe \\
\hline 0,28 & 1,17 & 0,16 & 0,016 & 0,012 & 0,0031 & Bal. \\
\hline
\end{tabular}

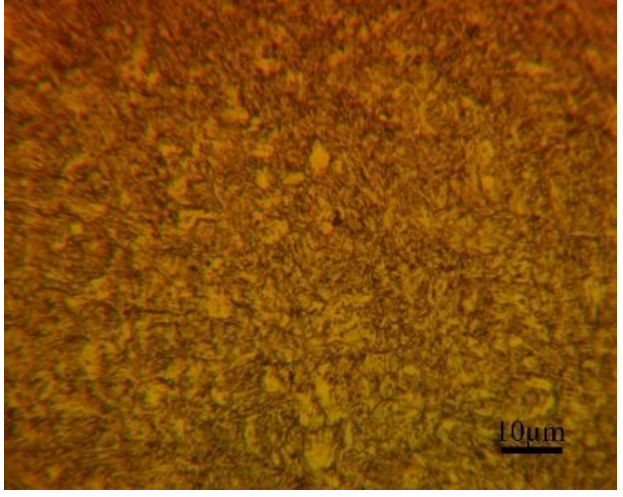

a)

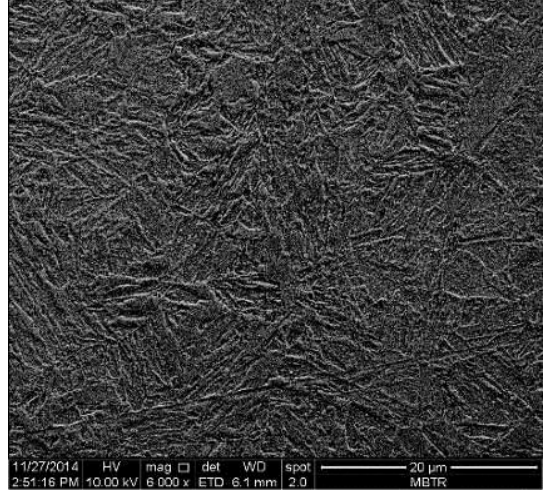

b)

Figura 3: Metalografías de la condición microestructural TR a) LM (x1000); b) SEM (x6000).

Análogamente, las Figura 4.a y b exhibe las metalografías ópticas y electrónicas de la condición microestructural DP, respectivamente. En la Figura 4.a la fase clara corresponde a la ferrita, mientras que la marrón a la martensita. En la Figura 4.b las fases comentadas se han indicado con $\alpha$ y $\alpha^{\prime}$, respectivamente. Ambas imágenes evidencian una estructura yuxtapuesta de $\alpha$ y $\alpha^{\prime}$ sin embargo, la mayor resolución de la Figura 4.b evidencia la presencia de precipitados en el interior de los granos ferríticos, los mismos estarían asociados a carboboruros de hierro no disueltos. La fracción en volumen de $\alpha^{\prime}$ es de $36 \pm 3 \%$. Este material alcanzó una dureza de $296 \pm 8 \mathrm{HV}$, un límite convencional al 0,2\% de 520MPa, una resistencia a la tracción de $945 \mathrm{MPa}$ y un alargamiento específico a rotura de 10,6\%. También, mediciones realizadas sobre probetas con su eje paralelo al sentido de laminación.

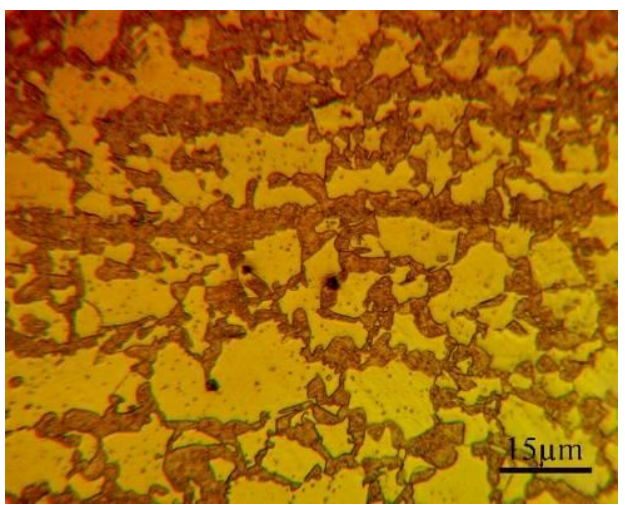

a)

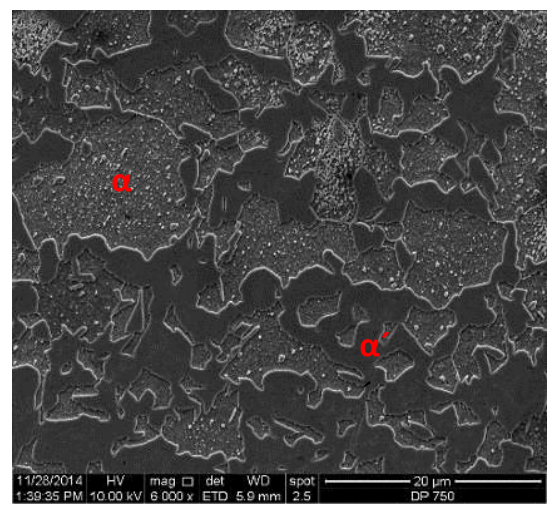

b)

Figura 4: Metalografías de la condición microestructural DP a) LM (x1000); b) SEM (x6000).

Las Figura 5.a y .b muestran fotografías de cara de las muestras TR y DP soldadas mediante PAW, correspondientemente. No se aprecian defectos y su aspecto superficial es bueno. En las Figura 6.a y .b se presentan macrografías correspondientes a cortes transversales de las uniones soldadas PAW+TR y PAW+DP, respectivamente. Sobre ellas se han superpuesto perfiles de microdureza Vickers tomados a lo largo de la línea media de los espesores.

En ambos casos puede verse ausencia de defectos internos (porosidad, socavados, fisuración), así como una penetración completa y adecuada conformación de raíz. Asimismo, pueden reconocerse las diferentes zonas que resultan tras la ejecución de la soldadura: Metal de Soldadura (MS), Zona Afectada por el Calor (ZAC) y Metal Base (MB).

En cuanto a los perfiles de microdureza, se observa que ambos resultan simétricos respecto a la línea central de la unión. Desde el MB la dureza disminuye monótonamente desde los 300HV hasta alcanzar un mínimo (Zona de Mínima Dureza, ZMD). La ZMD se extiende aproximadamente entre 3 y 4,3 mm para 
PAW+TR y PAW+DP, correspondientemente. Los valores de dureza mínimos registrados fueron de $242 \pm 4$ y 196 \pm 8 HV para PAW+TR y PAW+DP, respectivamente. Este mayor ablandamiento de la condición DP estaría asociado a la preexistencia de la fase ferrítica. Luego la dureza aumenta marcadamente hasta alcanzar valores de alrededor de $500 \mathrm{HV}$. Dichas zonas se extienden cerca de $2 \mathrm{~mm}$ para ambas configuraciones analizadas. Finalmente, en el MS la dureza disminuye oscilando alrededor de $350 \mathrm{HV}$, siendo el ancho del cordón de entre 2 y $3 \mathrm{~mm}$.

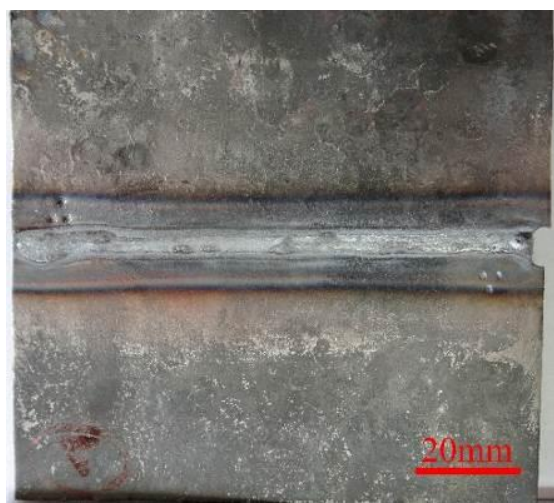

a)

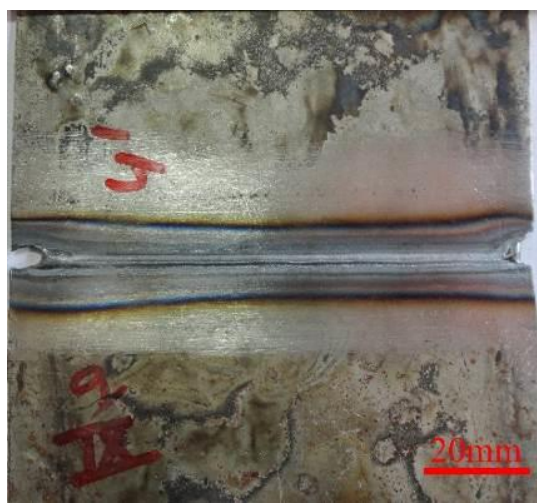

b)

Figura 5: Cordones de soldadura a) PAW+TR, b) PAW+DP.

a)

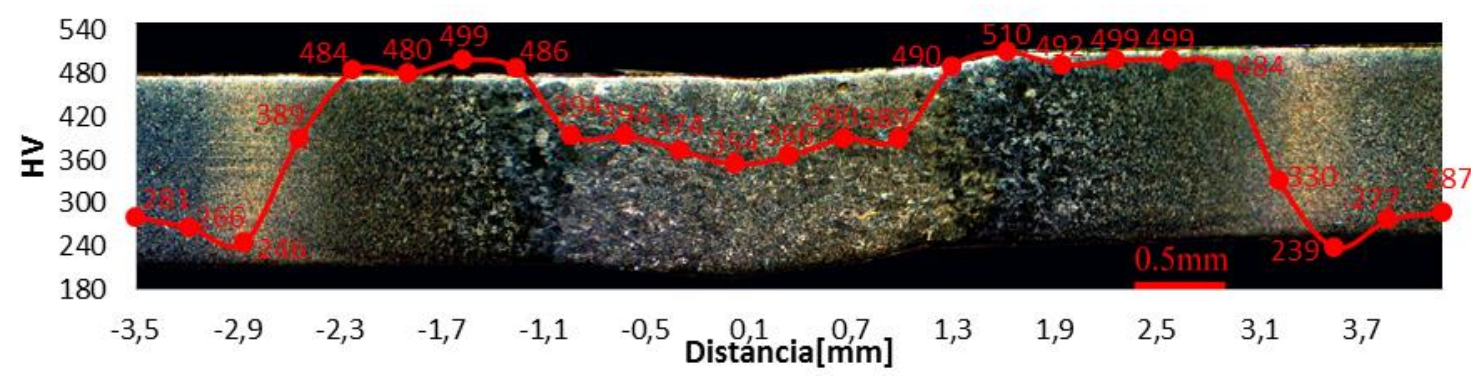

b)

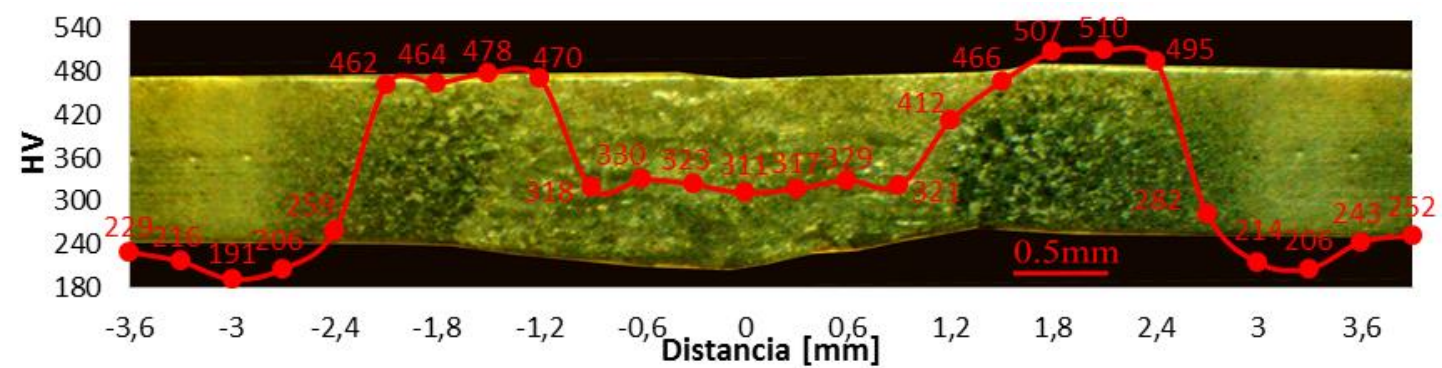

Figura 6: Macrografías y perfiles de microdureza a) PAW+TR; b) PAW+DP.

Las Figura 7 muestran la evolución microestructural consecuencia de los ciclos térmicos de soldadura impuestos durante la ejecución de las uniones. Desde el MB hacia el MS, se observa la zona del MB Revenido (MBR), dentro de la misma se reconoce la Zona Subcrítica (ZSC) como aquella donde la $\alpha^{\prime}$ se reviene a temperaturas cercanas, aunque inferiores, a $\mathrm{A}_{\mathrm{Cl}}$. Para el caso PAW+TR, se divisó una estructura ferrítica con una fina dispersión de precipitados, típica de martensita revenida a alta temperatura, mientras que, para PAW+DP, se observó una estructura similar aunque sectorizada consecuencia del arreglo de la $\alpha^{\prime}$ en paquetes. A continuación se presenta la Zona Intercrítica (ZIC), donde tiene lugar una austenización parcial y la $\alpha^{\prime}$ preexistente se reviene completamente, presentando una estructura de ferrita, carburos y martensita fresca. La ZIC de las uniones PAW+DP fue más cerrada que la observada para el caso de la PAW+TR, consecuencia de su estructura dual. Contiguamente se divisa la Zona Recristalizada Fina y más cercana al MS la Zona Recristalizada Gruesa $\left(Z_{\mathrm{f}}\right.$ y $\mathrm{ZR}_{\mathrm{g}}$, respectivamente). En ellas la austenización es total y en el enfriamiento, la austenita transformará por completo en martensita. Estas zonas se distinguen por el tamaño de grano, consecuencia de las temperaturas pico alcanzadas. Finalmente, en el MS se identificó una estructura de granos columnares, asociados a la solidificación, identificándose en distintas proporciones de ferrita acicular, poligonal y Widmanstätten, y martensita. 


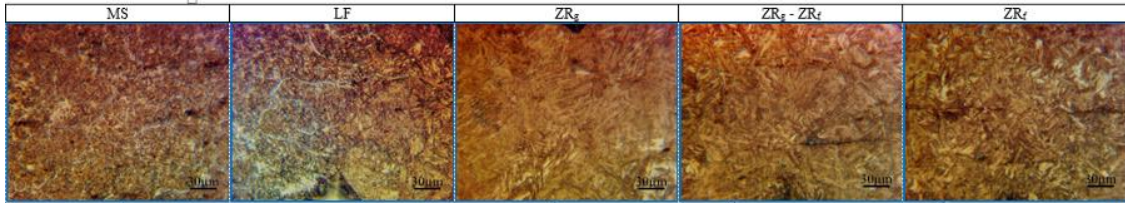

a)

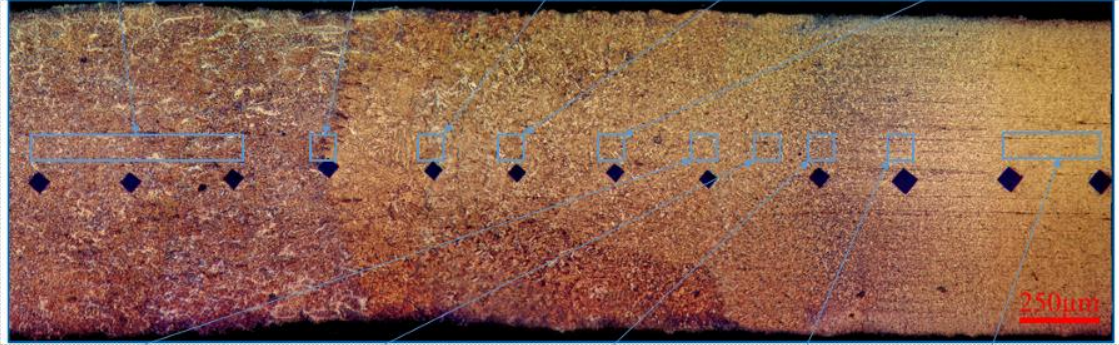

b)
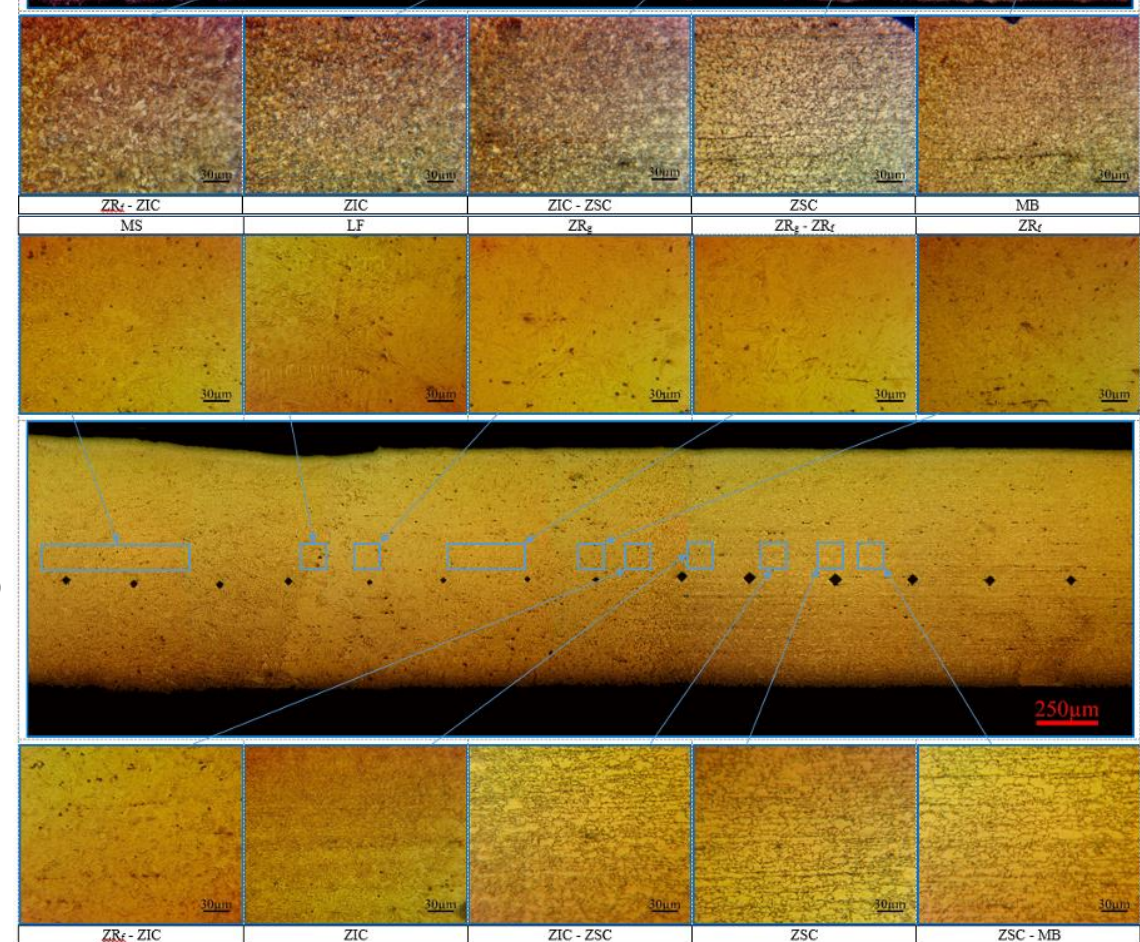

Figura 7: Evolución microestructural en la soldadura a) PAW+TR; b) PAW+DP.

La resistencia a la tracción de las uniones PAW+TR y PAW+DP fue de 915 y $792 \mathrm{MPa}$, respectivamente. En ambos casos la fractura se localizó en la Zona Blanda (ZB), se orientaron ortogonal al eje longitudinal de las probetas y sus superficies de fractura presentaron un aspecto dúctil. Estos resultados son consistentes con el mayor ablandamiento para la condición DP, como se mencionó anteriormente, siendo esa zona la que controla las propiedades en tracción de la junta.

El índice de resistencia $\left(\mathrm{I}_{\sigma}\right)$ se define como el cociente entre la resistencia a la tracción de la unión soldada y la resistencia a la tracción del $\mathrm{MB}$, y índice de dureza $\left(\mathrm{I}_{\mathrm{H}}\right)$ como la razón entre la dureza mínima medida en el corte transversal de la probeta soldada y la dureza del $\mathrm{MB}$, siendo los valores asociados para las uniones PAW+TR y PAW+DP de $\mathrm{I}_{\sigma}=0,97$ y $\mathrm{I}_{\mathrm{H}}=0,81$, y $\mathrm{I}_{\sigma}=0,84$ y $\mathrm{I}_{\mathrm{H}}=0,66$, respectivamente.

La Figura 8 presenta los datos de los ensayos de fatiga para ambos casos de estudio: PAW+TR y PAW+DP. Los puntos experimentales fueron ajustados potencialmente a fin de obtener las constantes de la ecuación de Basquin $\left(\mathrm{S}_{\mathrm{a}}=\mathrm{S}_{\text {ff }}^{\prime}(2 \mathrm{~N})^{\mathrm{b}}\right)$. A su vez, se determinaron los límites de fatiga (LF) a $10^{7}$ ciclos y los índices de fatiga $\left(\mathrm{I}_{\mathrm{f}}\right)$, determinados como el cociente entre el límite de fatiga y la resistencia a la tracción de la unión soldada. 


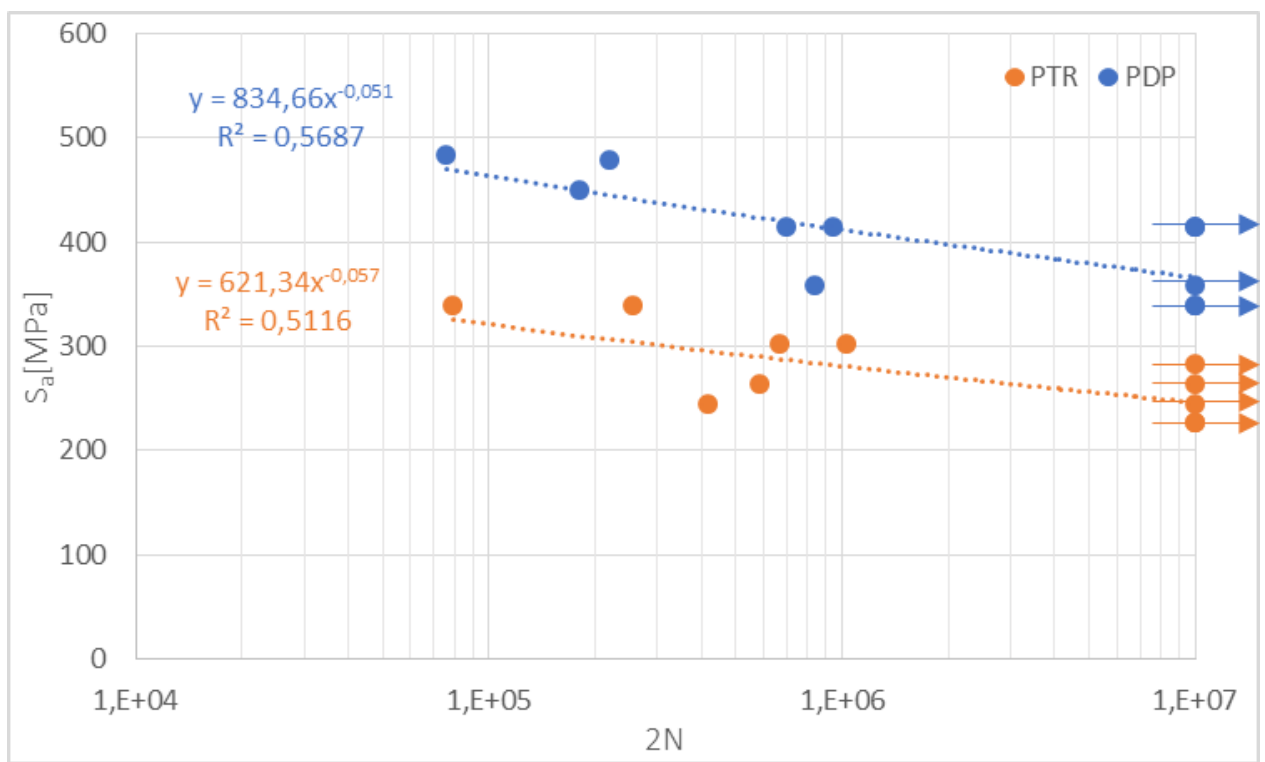

Figura 8: Curvas de fatiga de las uniones soldadas PAW+TR y PAW+DP.

La Tabla 3 resume los datos anteriormente indicados. Se observa un grado de correlación satisfactorio.

Tabla 3: Coeficientes de la ecuación de Basquin, Límites de fatiga e Índices de fatiga.

\begin{tabular}{l|l|l|l|l|l}
\hline \multirow{2}{*}{ DENOMINACIÓN } & \multicolumn{3}{|l|}{$\mathrm{S}_{\mathrm{a}}=\mathrm{S}_{\text {If }}^{\prime}(2 \mathrm{~N})^{\mathrm{b}}$} & \multirow{2}{*}{ LF } & \multirow{2}{*}{$\mathrm{I}_{\mathrm{f}}$} \\
\cline { 2 - 6 } & $\mathrm{S}_{\text {lf }}^{\prime}$ & $\mathrm{B}$ & $\mathrm{R}^{2}$ & & \\
\hline PAW+TR & 646,56 & $-0,057$ & 0,5116 & 226 & 0,25 \\
\hline PAW+DP & 864,76 & $-0,051$ & 0,5687 & 339 & 0,43 \\
\hline
\end{tabular}

Puede verse que la condición DP presenta un comportamiento a la fatiga superior que la condición TR en todo el intervalo de número de ciclos evaluado. Las fisuras dominantes de fatiga se localizaron en tres diferentes zonas: MS, ZB y MB. La Erro! Fonte de referência não encontrada.: Muestra correspondientes imágenes.

La Figura 10 muestra fractografías SEM de una muestra fracturada en la zona blanda, representativa de lo observado en los distintos ensayos ejecutados. En la Figura 10b se notan características típicas del fenómeno de fatiga como las estrías. No se detectaron tipologías propias asociadas a las diferentes ubicaciones de las fisuras dominantes de fatiga.

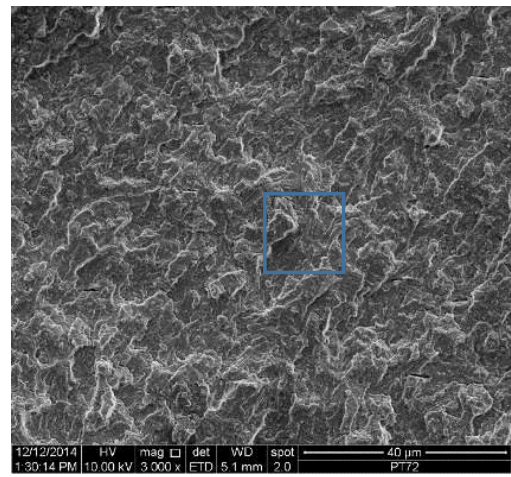

a)

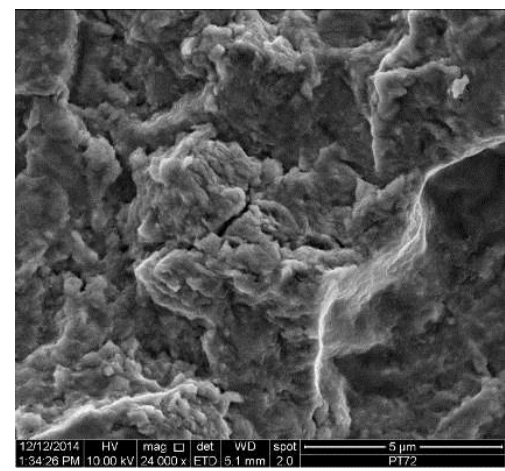

b)

Figura 9: Superficies de fractura de probetas ensayadas a fatiga - a) Zona blanda, b) magnificación del recuadro.

\section{DISCUSIÓN}

Partiendo de una misma aleación, tratamiento térmico mediante, se lograron dos configuraciones microestructurales diferentes. Las mismas lograron resistencias a la tracción semejantes pero la diferente estructura resultó en que la configuración DP exhibiese una deformación a rotura significativamente mayor respecto de 
la condición TR. Esto es característico en aceros de fase dual, elevada resistencia combinada con aceptable ductilidad. Sin embargo, se tiene también una disminución en su tensión de fluencia, siendo cerca del $60 \%$ de la tensión de fluencia del TR. De esta forma, se logra mancomunar elevadas resistencias y buena conformabilidad y, con ello, la posibilidad de producir piezas de geometría complicada y pequeños espesores [8].

La ejecución de las uniones soldadas altera la microestructura obtenida mediante el tratamiento térmico. Dicha modificación se pone de manifiesto a través del perfil de microdureza. Las regiones de máxima dureza se emparentan con la formación de martensita fresca. La disminución de dureza en la ZMD se asocian con el revenido de la $\alpha^{\prime}$ a diferentes niveles, siendo éste mayor cuanto más cerca se esté de la temperatura crítica inferior, alcanzada en la zona recristalizada [8]. Las desiguales durezas mínimas y extensiones de las distintas zonas se explicarían con la diferente fracción en volumen de martensita en cada material y la energía dispuesta por el proceso de soldadura. A igual energía, la intensidad del revenido será mayor a menor fracción en volumen de martensita. Ello estaría de acuerdo con la mayor extensión de la ZAC de la unión PAW+DP y la mayor dureza mínima y resistencia a la tracción de PAW+TR. Vale destacar los elevados índices de resistencia reportados.

En relación al comportamiento a la fatiga se nota que para el rango de números de ciclos analizado las uniones PAW+DP presentaron un mejor desempeño a fatiga. Si bien las pendientes de ambas curvas son semejantes, las amplitudes de tensiones toleradas por PAW+DP son mayores que las debidas a las uniones PAW+TR, para un dado número de ciclos a la falla. Más aún, el límite de fatiga de la junta PAW+DP es un $50 \%$ mayor que el LF de PAW+TR. Sin embargo, en fatiga de alto número de ciclos, conforme la resistencia estática del material aumenta, el desempeño a fatiga también mejora [9]. Por tanto, se esperaría que aquella unión con mayor dureza mínima presentase un mejor comportamiento a fatiga y que todas las uniones fallasen por la ZB, lo que no fue sistemáticamente observado.

En la Figura 11 se identifica la ubicación de la fractura de los distintos ensayos realizados. No se observa una tendencia clara en ese sentido.

Las fisuras sucedidas en el MB se asociaron con defectos introducidos durante el proceso de fabricación de las probetas (rayados, rebabas de mecanizado) por tanto, se entienden no significativas. Para los dos tipos de junta las fallas que acontecieron en el MS tuvieron lugar en todo el rango de $\mathrm{N}$ y $\mathrm{S}_{\mathrm{a}}$, en ningún caso se agruparon en forma particular. Esta dispersión de datos se relacionó con concentradores geométricos de tensión debidos a desalineaciones de las chapas, rugosidad del cordón y reducciones de la sección en los cordones de soldadura. No obstante, las fallas en las ZB sí se presentan agrupadas y con la contradicción anteriormente indicada, las ZB de menor dureza mínima (PAW+DP) toleraron mayores amplitudes de tensión, aunque su vida a fatiga fueron sensiblemente menores a las de mayor dureza mínima (PAW+TR).

En general, la vida a la fatiga de uniones soldadas se ve controlada fundamentalmente por los concentradores geométricos de tensión, afectados a su vez por el campo de tensiones residuales, siendo los aspectos metalúrgicos de menor incidencia en dicho comportamiento a la fatiga [10]. Sin embargo, en esta situación, donde la fractura se ubica en la ZAC, donde no se tienen concentradores y dado que las tensiones residuales han sido relevadas durante el corte de las probetas soldadas, una posible explicación a este comportamiento podría estar emparentada con la evolución microestructural que acontece en la ZB.

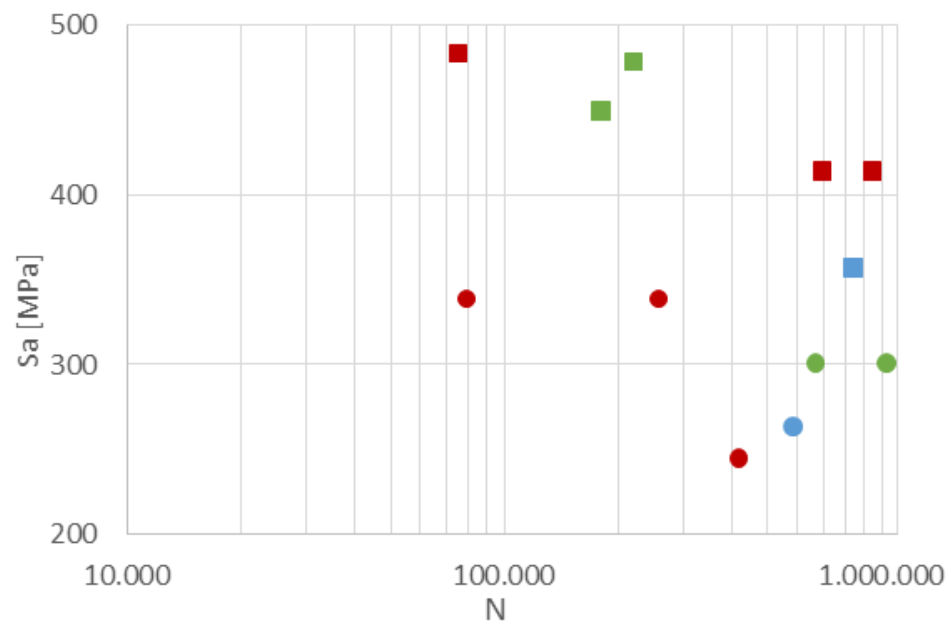

Figura 10: Pares ordenados $\left(\mathrm{S}_{\mathrm{a}} ; \mathrm{N}\right)$ vs. localización de la fisura; $\bigcirc$ PAW+TR; $\square$ PAW+DP; $\bigcirc$ Metal de Soldadura; Zona Blanda; Material Base. 
Dado que la composición química de ambas uniones es la misma, la estructura en las zonas recristalizadas y en el metal de soldadura es semejante en ambos casos, siendo diferentes solo en las zonas ZIC y ZSC (Zona Blanda). En la Figura 11 se muestran detalles de las estructuras que resultan en la ZB de ambas uniones.

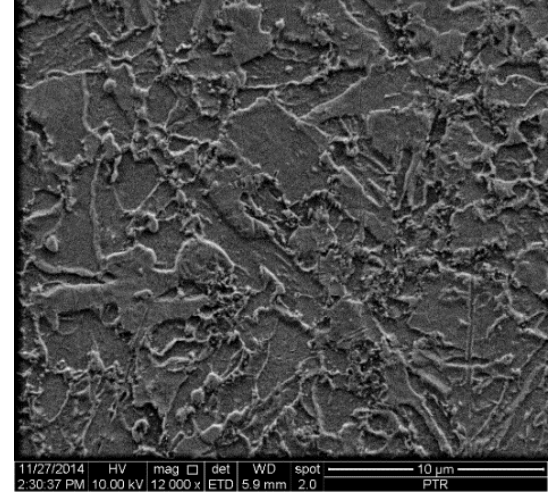

a)

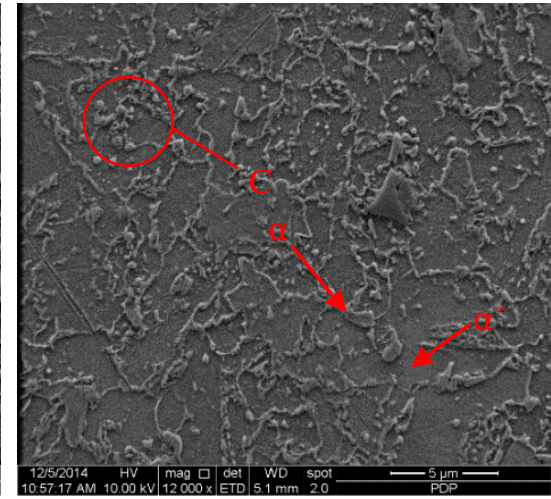

b)

Figura 11: Microscopía electrónica de barrido. Zona Blanda a) PAW+TR; b) PAW+DP.

Puede verse que presentan diferencias que podrían ser relevantes. Para el caso PAW+DP se tiene una estructura con una distribución más fina de precipitados (carboboruros de hierro) y $\alpha^{\prime}$ embebidas en la matriz más blanda de ferrita, con restos de martensita revenida. La diferente distribución, morfología y fracción en volumen de las fases duras se entienden como los factores que podrían explicar que la acumulación de daño que se impone con la aplicación reiterada de carga sea retrasada en la unión de menor dureza mínima (PAW+DP). Esto último podría estar relacionado con las observaciones realizadas precedentemente respecto de la mejor respuesta a fatiga de las microestructuras DP, donde se atribuye dicho comportamiento a una compleja interacción de la estructura de dislocaciones en las inmediaciones de las interfases ferritamartensita [8]. Sin embargo, se requiere profundizar en la caracterización de dichas zonas a fin de desarrollar algún modelo que explique el comportamiento observado.

\section{CONCLUSIONES}

$\checkmark$ Se obtuvieron dos configuraciones microestructurales diferentes (TR y DP) de similar resistencia a la tracción $(950 \mathrm{MPa})$ partiendo de un acero microaleado al boro en chapa de $1 \mathrm{~mm}$ de espesor.

$\checkmark$ Se generaron uniones soldadas mediante el proceso PAW para ambas configuraciones microestructurales, empleando los mismos parámetros de soldadura. Dichas uniones presentaron una buena calidad, libres de defectos macroscópicos, observándose una evolución microestructural asociada a los ciclos térmicos impuestos.

$\checkmark \quad$ Las uniones PAW+DP presentaron una degradación mayor que las PAW+TR, alcanzando una microdureza mínima de $196 \mathrm{HV}$ en lugar de $242 \mathrm{HV}$ como las PAW+TR. Las eficiencias de junta fueron elevadas de 0,81 y 0,84 para PAW+TR y PAW+DP, respectivamente.

$\checkmark \quad$ La vida a fatiga y límite de fatiga de las uniones PAW+DP fue superior que las debidas a las juntas PAW+TR, para todo el rango de ciclos estudiado. Ello podría estar asociado con la diferente microestructura que tiene lugar en la zona blanda. Sin embargo debe profundizarse la caracterización de dichas zonas para lograr una mejor comprensión de los fenómenos actuantes.

\section{AGRADECIMIENTOS}

Los autores de este trabajo desean agradecer por el apoyo a INTI-Mecánica, la Universidad de Buenos Aires Air Liquide Argentina y Ternium-Siderar.

\section{BIBLIOGRAFÍA}

[1] LARA, A. et al., "Effect of the cutting process on the fatigue behavior of press hardened and high strength dual phase steels", Journal of Materials Processing Technology, v. 213, n. 11, pp. 1908-1919, Nov. 2013. 
[2] DAVENPORT, A. T., Formable HSLA and Dual-Phase Steels: Proceedings of a Symposium, ilustrada, Califronia, Metallurgical Society of AIME, 1979.

[3] SANCHEZ, N. et al., "Obtención de aceros Dual Phase (DP) a partir de acero microaleado al boro", Tercer Congreso Argentino de Ingeniería Mecánica, Oct. 2012.

[4] KEELE, R., KIMICHI, M., Advanced High-Strength Steels: Application Guidelines, 5 ed., World Auto Steel, 2014.

[5] SCOTTI, A., REIS, R.P., Fundamentos e práctica da soldagem a plasma, 1 ed., São Paulo, Artliber Editora Ltda., 2007.

[6] FARABI, N. et al., "Fatigue properties of laser welded dual-phase steel joints", Procedia Engineering, v. 2, n. 1, pp. 835-843, Mar. 2010.

[7] RADAJ, D. et al., "Recent developments in local concepts of fatigue assessment of welded joints", International Journal of Fatigue, v. 31, n. 1, pp. 2-11, Ene. 2009.

[8] TAYANC, M. et al., "The effect of carbon content on fatigue strength of dual-phase steels", Materials and Design, v. 28, n. 1, pp. 1827-1835, Abr. 2006.

[9] DIETER, G. E., Mechanical Metallurgy, 3 ed., London, McGraw-Hill, 1988.

[10] RADAJ, D., Design and analysis of fatigue resistant welded structures, 1 ed., Cambridge, Abingtong Publishing, 1990. 